\title{
lonospheric scintillation intensity fading characteristics and GPS receiver tracking performance at low latitudes
}

\author{
Kai Guo ${ }^{1} \cdot$ Marcio Aquino $^{1} \cdot$ Sreeja Vadakke Veettil ${ }^{1}$
}

Received: 19 September 2018 / Accepted: 13 February 2019 / Published online: 27 February 2019

(c) The Author(s) 2019

\begin{abstract}
Ionospheric scintillation refers to rapid fluctuations in signal amplitude/phase when radio signals propagate through irregularities in the ionosphere. The occurrence of ionospheric scintillation can severely degrade the Global Navigation Satellite System (GNSS) receiver tracking loop performance, with consequential effects on positioning. Under strong scintillation conditions, receivers can even lose lock on satellites, which poses serious threats to safety-critical GNSS applications and precise positioning. The characteristics of intensity fading on Global Positioning System (GPS) L1 C/A signals during the peak of the last solar cycle at the low latitude station of Presidente Prudente (Lat. 22.12 ${ }^{\circ}$ S, Long. $51.41^{\circ} \mathrm{W}$, Magnetic Lat. $12.74^{\circ} \mathrm{S}$ ) are investigated. The results show that the occurrence of scintillation at this station is extremely frequent. An analysis of the fading events revealed an inverse relationship between fading depth and duration. Mathematical models are built to investigate and explain the statistical relationship between intensity fading and the commonly used amplitude scintillation index $S 4$. Then the GPS receiver tracking loop performance is studied in relation to fading. A conclusion can be drawn that both fading depth and duration can affect the tracking loop performance, but the tracking error variance is more strongly related to fading speed, defined as the ratio of fading depth to fading duration. The proposed study is of great significance for better understanding the ionospheric scintillation intensity fading characteristics at low latitudes. It can also contribute to the research on the effects of scintillation on GNSS as well as support the design and development of scintillation robust GNSS receivers.
\end{abstract}

Keywords Ionospheric scintillation · Global Positioning System · Intensity fading $\cdot$ Tracking performance $\cdot$ Amplitude scintillation

\section{Introduction}

The ionosphere is the ionized part of the earth's atmosphere in which the number of free ions and electrons is large enough to affect the propagation of radio frequency $(\mathrm{RF})$ signals. At low latitudes, ionospheric plasma density irregularities are formed due to the Fountain effect (Davies 1990; Yeh and Liu 1982), which results in a phenomenon known as scintillation, characterized by rapid signal amplitude/phase fluctuations when RF signals pass through the irregularities.

Scintillation has attracted extensive research interests in the past several years. Researchers such as Basu et al. (1988), Fortes et al. (2015) and Kai et al. (2017) found that

\section{Kai Guo}

kai.guo@nottingham.ac.uk

1 Nottingham Geospatial Institute, University of Nottingham, Nottingham, UK the occurrence of scintillation is modulated by solar and geomagnetic activity. During the peaks of the solar cycles and during geomagnetic storms, the ionosphere turbulent plasma becomes more active and severely influences the propagation of radio signals. Ionospheric scintillation occurrence also presents strong temporal and spatial dependencies. It varies from day to day and is more frequent during the post sunset hours at low latitudes. Additionally, during vernal and autumnal equinoxes, there is a higher probability of scintillation occurrence. For the global distribution, Aarons (1982) and Basu et al. (1988) pointed out that scintillation is more likely to occur both in the auroral to polar region and in the equatorial region.

Scintillation occurrence can affect the quality of the Global Navigation Satellite System (GNSS) signals, thus degrading the GNSS receiver tracking loop performance and consequently positioning accuracy. The effects of scintillation on the GNSS receiver performance have been widely 
investigated. Sreeja et al. (2012) analyzed the correlation between scintillation levels and GNSS receiver tracking performance for both Global Positioning System (GPS) signals, L1C/A and L2C, as well as GLONASS L1 and L2 signals. The results showed that the signals with lower transmitting frequency are more affected under scintillation and the correlation between scintillation levels and the Phase Locked Loop (PLL) tracking jitter can be represented by a quadratic function. The performance of several GPS receivers under severe scintillation conditions was tested by Groves et al. (2000). They concluded that the performance of the receivers varies, and thus the modeling of scintillation effects should be receiver-specific. To theoretically quantify the scintillation effects on a GPS receiver, Conker et al. (2003) and Hegarty et al. (2001) built statistical models to estimate the tracking error variance. Then tracking jitter maps can be developed (Sreeja et al. 2011) and exploited to modify the least squares stochastic model used to estimate position to mitigate scintillation effects, consequently improving the GNSS positioning accuracy (Aquino et al. 2009). Furthermore, loss of lock on satellites or cycle slips may occur from time to time due to scintillation, posing serious threats to safety-critical GNSS applications and precise positioning.

Extensive research effort has been placed on investigating GNSS signal intensity fading due to scintillation. Moraes et al. (2012) investigated the intensity fading characteristics based on one month's scintillation data collected at low latitudes and built the relationship between fading duration and the probability of occurrence of cycle slips. They concluded that the performance of a GPS receiver with $C / N_{0}$ threshold around or higher than $30 \mathrm{~dB} \mathrm{~Hz}$ could be severely influenced by deep fading. On the other hand, Jiao et al. (2016) studied the scintillation amplitude fading characteristics on GPS L1, L2C and L5 signals at the equatorial region. The results showed that the fading rarely occurs on all GPS bands at the same time. Moreover, based on the intermediate frequency (IF) data and using a commercial software receiver, $45 \mathrm{~min}$ of $50 \mathrm{~Hz} C / N_{0}$ data were analyzed by Seo et al. (2016) to characterize the signal fading due to scintillation. A fading duration model was also built, which was beneficial for designing an aviation receiver with short reacquisition time to counter the adverse effects of scintillation. However, these studies were based on data sets which did not include many severe scintillation events and were collected within a short period. Additionally, previous studies mostly ignore the direct influence of signal fading on receiver performance, where the relationship between fading and tracking loop performance was not investigated.

The GPS signal intensity fading due to scintillation over 3 months during the peak of solar cycle 24 at Presidente Prudente in Brazil, a low latitude station within one of the worst global sectors affected by scintillation, is investigated. The main issues of interest in this study are: (1) characterizing the scintillation intensity fading and investigating the relationship between fading depth, duration and the amplitude scintillation index, $S 4$; (2) analysing for the first time the effects of intensity fading on receiver tracking performance. The scintillation indices and intensity fading characterization are introduced next, followed by an explanation of PLL tracking errors and the data sets analyzed in this study. The results and discussion are presented subsequently, followed by the conclusions and remarks of this study.

\section{Amplitude scintillation and intensity fading characterizing}

Ionospheric scintillation is normally categorized as amplitude and phase scintillation, referring to the sharp fluctuation in amplitude/intensity and carrier phase measurements, respectively. As the fading mainly refers to fluctuations in intensity, and as amplitude scintillation is more frequent and severer than phase scintillation at low latitudes, only amplitude scintillation is introduced in this analysis. The intensity of amplitude scintillation is commonly indicated by $S 4$, which is the standard deviation of the normalized signal power over $1 \mathrm{~min}$, given by (Van Dierendonck et al. 1993; Van Dierendonck and ArbesserRastburg 2004),

$S 4=\sqrt{\frac{\left\langle P_{\text {det }}^{2}\right\rangle-\left\langle P_{\text {det }}^{2}\right\rangle}{\left\langle P_{\text {det }}^{2}\right\rangle}}$,

where $P_{\text {det }}$ is the detrended signal intensity measurement and $\langle\cdot\rangle$ denotes mathematical average over $60 \mathrm{~s}$. The signal detrending and the calculation of $P_{\operatorname{det}}$ is to be explained in detail next.

The focus of this study is to characterize scintillationcaused intensity fadings and to investigate the effect of these fadings on GPS receiver performance. Therefore, other fluctuations and noise compounded into the signal, such as lowfrequency range variation caused by satellite motion, satellite clocks, receiver clock, tropospheric delay, multipath and thermal noise, should be removed to avoid contamination of the actual scintillation data. This process is called measurement detrending (Mushini et al. 2012; Van Dierendonck et al. 1993). To remove the multipath effects on signal intensity measurements, the satellite/receiver links with an elevation lower than $30^{\circ}$ are ignored.

The scintillation monitoring receiver used in this study can output $50 \mathrm{~Hz}$ post-correlation In-phase $\left(I_{\text {corr }}\right)$ and Quadraphase $\left(Q_{\text {corr }}\right)$ measurements. Thus, the signal intensity measurement $P$ is calculated by:

$P=I_{\text {corr }}^{2}+Q_{\text {corr }}^{2}$.

As previously mentioned, low-frequency signal intensity variation contributes to the noise in scintillation data. 
To detrend this part of the noise, the intensity measurements are first passed through a low-pass filter to obtain the intensity trend, denoted as $P_{\text {trend }}$. Then $P$ is normalized by 60 -s averaged outputs of the filter, i.e. $P_{\text {trend }}$, as in the following equation (Van Dierendonck et al. 1993; Van Dierendonck and Arbesser-Rastburg 2004):

$P_{\text {det }}=\frac{P}{\left\langle P_{\text {trend }}\right\rangle_{60 \mathrm{~s}}}$.

A sixth-order Butterworth low-pass filter with a cutoff frequency of $0.1 \mathrm{~Hz}$ is used to detrend the intensity measurements. After detrending, the value of $P_{\text {det }}$ will fluctuate around $1(0 \mathrm{~dB})$.

Figure 1 shows an example of the intensity measurement detrending process based on GPS L1 C/A signal for PRN 18 observed between 00:00 and 02:20 UTC on December 11, 2014. During this period, scintillation events were captured as shown in the top panel. The scintillation caused significant fluctuations in intensity. Apart from these fluctuations, the signal intensity also varies with time due to satellite motion as denoted by the red line in the middle panel. This trend is due to satellite motion and was further removed through the process of intensity normalization. In the bottom panel, the detrended intensity fluctuates around the value of $0 \mathrm{~dB}$. Comparing the top and bottom panels, it can be observed that the extent of fluctuation is substantially modulated by $S 4$.

The signal intensity fadings due to scintillation are then detected based on the detrended intensity measurements. The fading duration, $t_{\mathrm{f}}$, is defined as the time difference between the beginning and end of the fading, while the fading depth, $d_{\mathrm{f}}$, is defined as the minimum intensity within the fading (Akala et al. 2012; Jiao et al. 2016; Moraes et al. 2012). Figure 2 presents examples of the intensity fadings captured by the receiver at the station. The depth of the first fading in the figure is around $-40 \mathrm{~dB}$ and the duration is around $1.2 \mathrm{~s}$. The threshold used to define and detect intensity fading varies in the literature. A threshold of $-5 \mathrm{~dB}$ is applied in this study, as this threshold can clearly distinguish between the fading caused by scintillation and ambient noise. It also guarantees enough number of samples for different scintillation levels to support the statistical analysis. The selection of fading threshold will be further explained. It is worth mentioning that although the signal intensity measurements are detrended, there is still some noise left in the measurements. Therefore, a threshold of $0.1 \mathrm{~s}$ was also applied to select the detected fading events. In other words, only intensity fadings lasting longer than $0.1 \mathrm{~s}$ were considered.
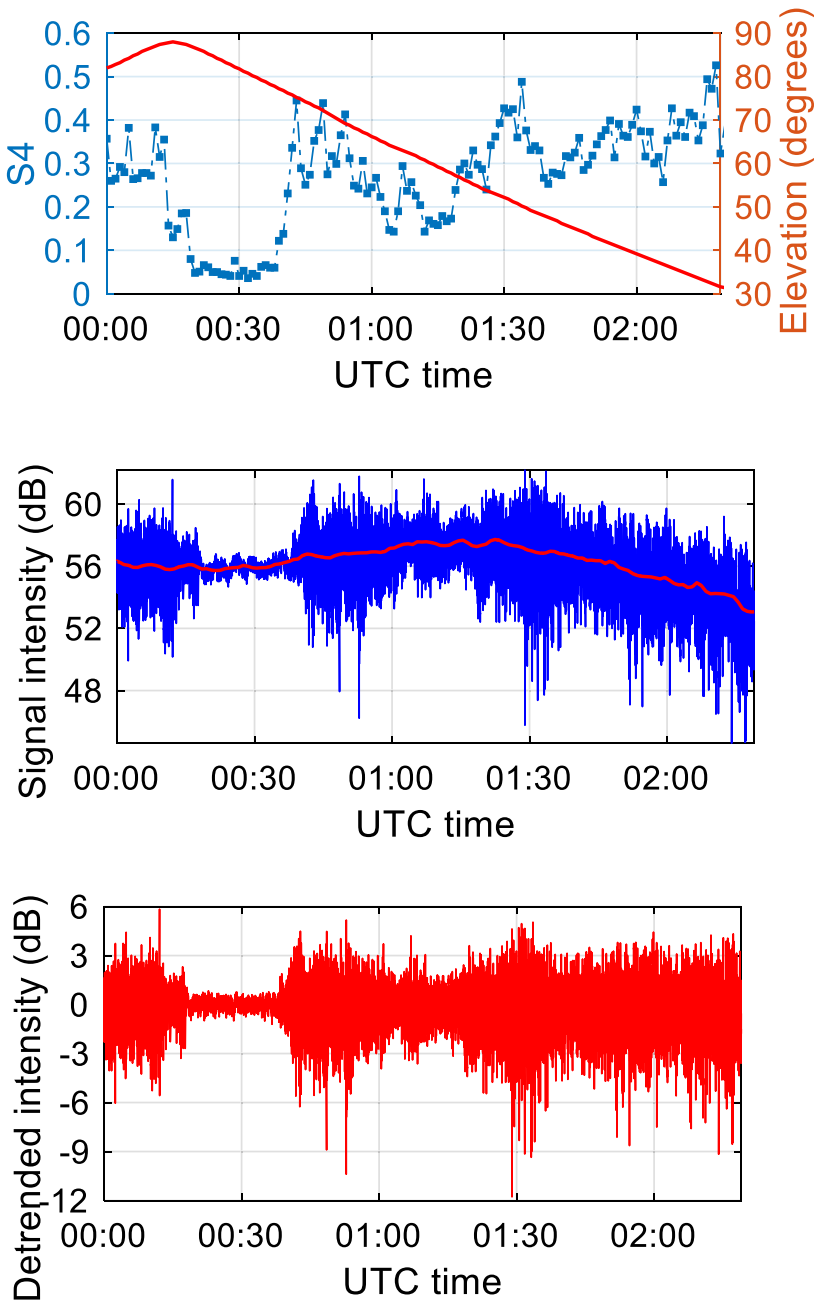

Fig. 1 Intensity measurement detrending on GPS L1 C/A signal for PRN 18 observed between 00:00 and 02:20 on December 11, 2014 at Presidente Prudente. Figures, respectively, show the variations in $S 4$ and elevation (top), measured signal intensity and trend (middle) and detrended signal intensity (bottom) as a function of time

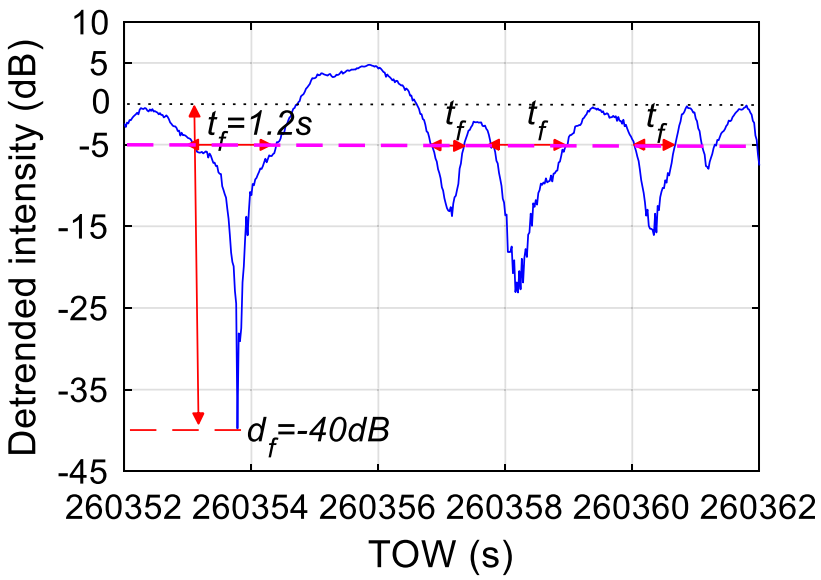

Fig. 2 A demonstration of fading depth and duration on detrended signal intensity with a threshold of $-5 \mathrm{~dB}$ 


\section{PLL tracking error variance}

The satellite signal tracking is accomplished by the PLL and delay locked loop (DLL) in the receiver. In the PLL, the tracking error is output by the carrier loop discriminator and is widely used to assess the receiver performance under different scintillation conditions (Forte 2012; Hegarty et al. 2001; Sreeja et al. 2012). A thirdorder tracking loop with a discriminator of an arctangent function is implemented in the ionospheric scintillation monitor receiver (ISMR). Therefore, the tracking error for the GPS L1 C/A signal is given by (Kaplan and Hegarty 2005):

PLL tracking error $(\mathrm{rad})=\operatorname{atan}\left(\frac{Q_{\text {corr }}}{I_{\text {corr }}}\right)$.

The PLL tracking error variance is given by:

$\sigma_{\mathrm{PLL}}^{2}\left(\operatorname{rad}^{2}\right)=[\operatorname{std}(\mathrm{PLL} \text { tracking errors })]^{2}$.

With the tracking loop performance indicated by tracking error variance, correlation analysis is implemented to investigate the relationship between fading duration, depth and PLL tracking performance.

\section{Data set}

The GPS scintillation data were collected by an ISMR installed at Presidente Prudente (lat. $22.12^{\circ} \mathrm{S}$, long. $51.41^{\circ} \mathrm{W}$, magnetic lat. $12.74^{\circ} \mathrm{S}$ ) in the frame of the CIGALA/CALIBRA projects funded by the EC 7 Framework Program (Vani et al. 2017). Scintillation over the
Brazilian territory is particularly active as this sector is close to both the equatorial ionization anomaly (EIA) and the South Atlantic Magnetic Anomaly (SAMA) (Spogli et al. 2013).

The ISMR at this station is a Septentrio PolaRxS Pro receiver, which is a specialized multi-frequency, multiconstellation receiver for ionospheric monitoring and space weather research. The amplitude and phase samples are generated at a frequency of $50 \mathrm{~Hz}$ to calculate the scintillation indices along with other output parameters such as $C / N_{0}$, satellite lock time, elevation, azimuth, spectral parameters, and total electron content (TEC). It is worth mentioning that the PolaRxS receiver enables users to configure the tracking loop parameters. In this analysis, the PLL bandwidth is configured as $15 \mathrm{~Hz}$ and the coherent integration time is set to $10 \mathrm{~ms}$.

Scintillation data recorded on the GPS L1 C/A signal from October 1 to December 31, 2014 was selected to carry out the study. The period was chosen according to the solar activity, which peaked in 2014 , and data availability. As the scintillation occurs during night-time, the data collected during daytime were not considered. Thus, a total of $1068 \mathrm{~h}$ of scintillation data were analyzed in this study.

\section{Overviews of the scintillation and intensity fading}

In this section, the daily amplitude scintillation index was statistically analyzed and signal intensity fadings with different depths and duration were counted, to give an overall view of the ionospheric scintillation at low latitudes. The relationship between fading depth and duration was then investigated.

Figure 3 presents the daily occurrence of various levels of amplitude scintillation observed at Presidente Prudente over the 3 months. The statistics are based on the scintillation
Fig. 3 Daily occurrence for various levels of amplitude scintillation observed at Presidente Prudente from October 1 to December 31, 2014

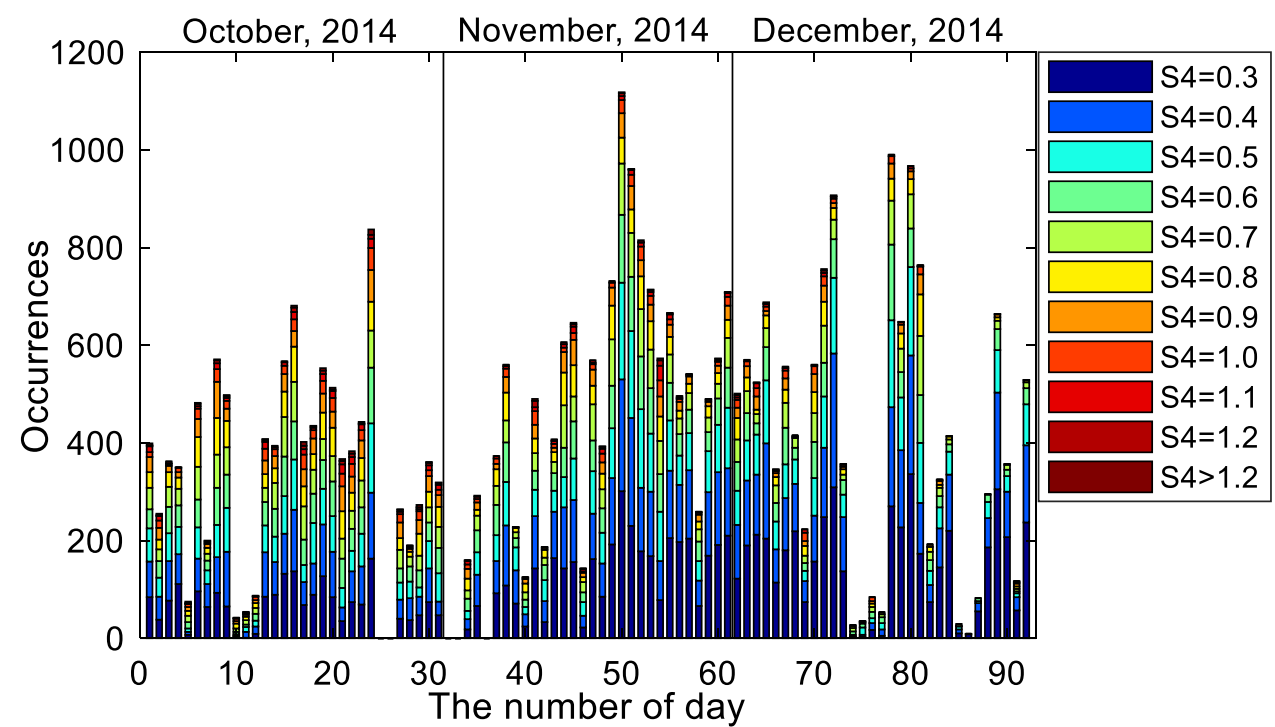




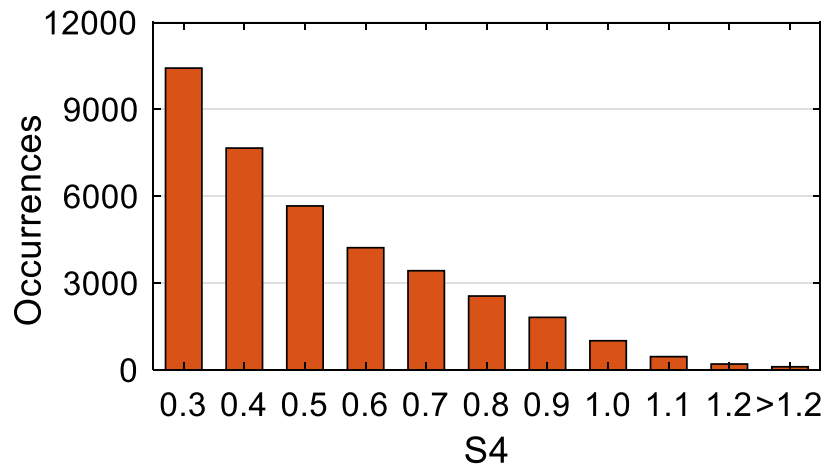

Fig. 4 Occurrence of scintillation in relation to $S 4$ over the period from October 1 to December 31, 2014

index $S 4$ computed over each 60 s using the detrended signal intensity measurements. It can be seen from the figure that strong scintillation, with $S 4>0.7$, occurs almost every day and that the value of $S 4$ can reach as much as 1.2. Additionally, large day-to-day variability of scintillation occurrence can also be observed. On average, the occurrence of scintillation is lower than 500 per day. However, on November 19 , 2014, the number of occurrences reached a value of 1118 which is a particularly extreme situation. Due to the unavailability of scintillation data on October 25, 26 and November $1,2,5$, the scintillation occurrences for these days are not shown.

Figure 4 illustrates the occurrence of scintillation in relation to $S 4$. It is obvious that the occurrence of scintillation is mostly characterized by values of $S 4$ between 0.3 and 0.9 , with fewer occurrences when $S 4$ increases further. However, although the occurrence of scintillation with $S 4>1.0$ is relatively low, these scintillation events may severely degrade the receiver tracking performance and should be considered separately.

Using $-5 \mathrm{~dB}$ as the threshold, a total number of 144,891 fading events were detected. The fading occurrences in relation to $S 4$ are shown in the top panel of Fig. 5. The occurrence of fading peaks when $S 4=0.8$, although the occurrence of scintillation at this level is not very high (see Fig. 4). Meanwhile, the fading occurrence increases significantly from 26 for $S 4=0.2$ to 1067 for $S 4=0.3$ when scintillation is considered to occur. Thus, it can be concluded that the value of $-5 \mathrm{~dB}$ can clearly and effectively distinguish between intensity fading caused by scintillation and signal fluctuation caused by ambient noise. The number of detected fadings using $-10 \mathrm{~dB}$ as the threshold is also shown in the bottom panel. It can be seen that even when $S 4=0.4$, the number of detected fadings is only 144, which is not enough for statistical analysis. This may be due to the fact that the threshold of -10 $\mathrm{dB}$ is not appropriate and ignores plenty of intensity fading caused by weak scintillation. Consequently, a threshold of
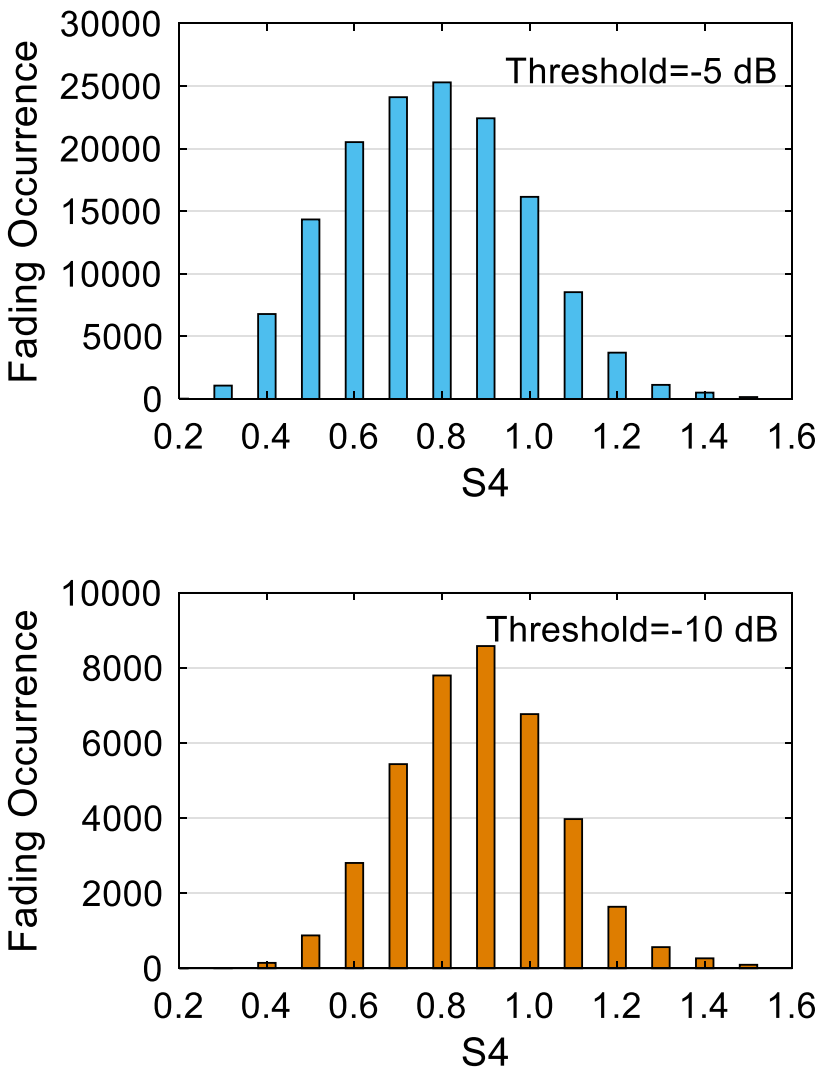

Fig. 5 Fading occurrence in relation to $S 4$ detected using thresholds of $-5 \mathrm{~dB}$ (top) and $-10 \mathrm{~dB}$ (bottom)

$-5 \mathrm{~dB}$ is applied in this study to give a general understanding of the scintillation intensity fading. The relationship between fading depth, duration and scintillation levels is investigated hereafter.

The distribution of the detected fading as a function of fading depth is demonstrated in the top panel of Fig. 6. The number of fading events decreases rapidly as the fading becomes deeper. Most of the fadings are between -5 and $-15 \mathrm{~dB}$. Similarly, the bottom panel presents the distribution of fadings in relation to fading duration. As shown in the figure, most of the fadings are within $1 \mathrm{~s}$. The occurrence of fadings decreases dramatically with the increase of fading duration.

To illustrate the relationship between fading depth and duration, Fig. 7 presents a scatter plot based on all the detected fadings. Although the average fading depth and duration are $-10.39 \mathrm{~dB}$ and $0.7291 \mathrm{~s}$, respectively, the fading can be as deep as $-58 \mathrm{~dB}$ and last as long as $31 \mathrm{~s}$. Additionally, the fading depth and duration tend to present an inverse relationship. In other words, for fadings with depth lower than $-40 \mathrm{~dB}$, the duration is usually less than around $3 \mathrm{~s}$. While for fadings with longer duration, it tends to be not too deep. There are a few fadings with long duration which are extremely deep. 


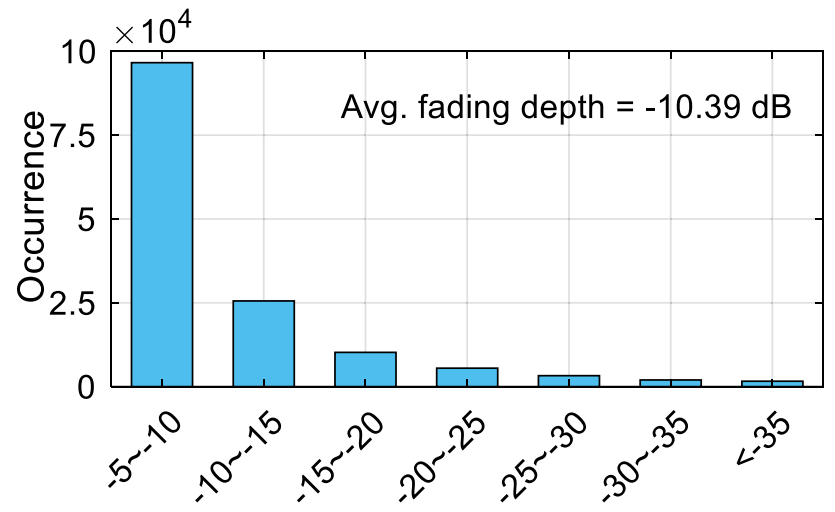

Fading depth(dB)

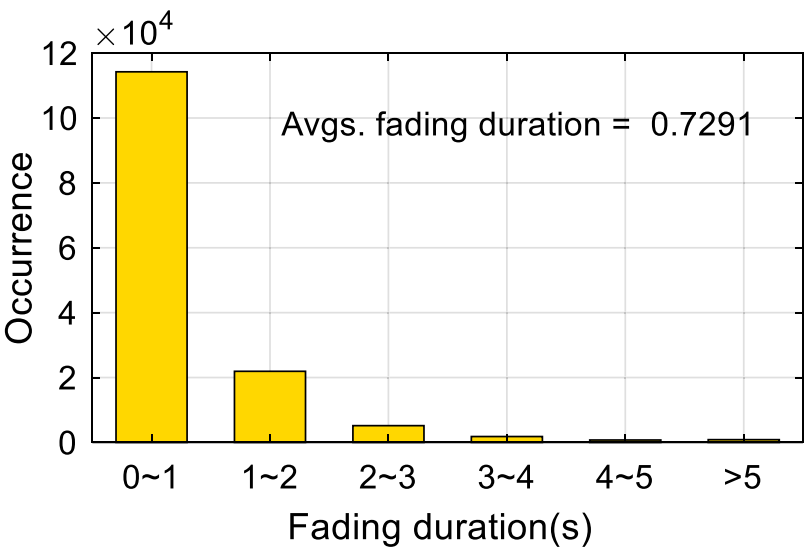

Fig. 6 Distribution of detected fadings in relation to fading depth (top) and fading duration (bottom)

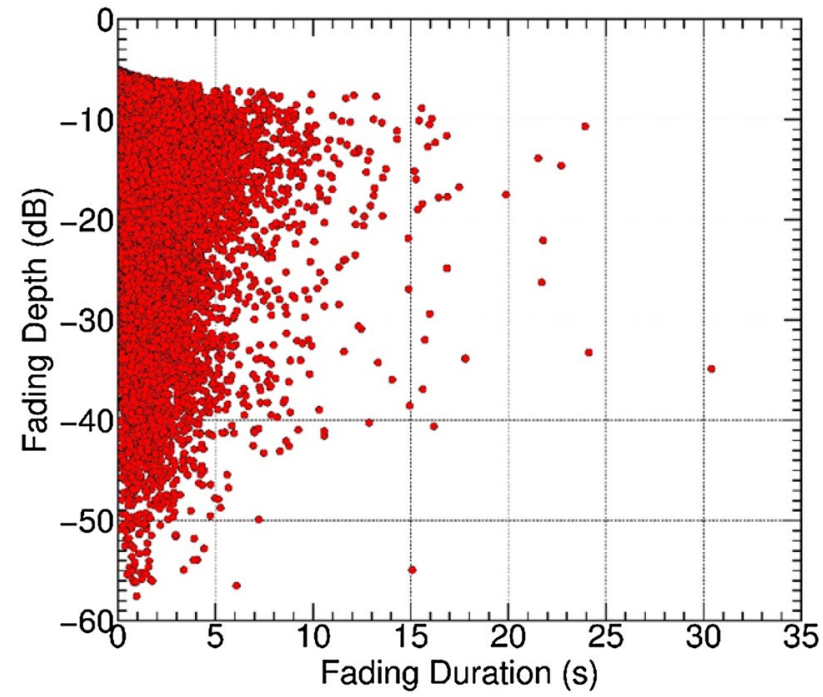

Fig. 7 Relationship between fading depth and duration for all detected fadings

\section{Scintillation intensity fading and S4}

This section investigates the relationship between amplitude scintillation levels and signal intensity fading. The study is carried out from two perspectives, i.e. (1) analyzing the overall distribution of fading depth and duration in relation to $S 4$; (2) analyzing the average fading depth, duration and occurrence based on every single scintillation event. Figure 8 shows the distribution of fading depth and duration as a function of $S 4$. Every dot in the figure corresponds to a detected intensity fading. In the top panel, it can be seen that intensity fadings higher than $-20 \mathrm{~dB}$ can occur under all scintillation levels. Even for the very strong scintillation levels, fadings that are not too deep may occur. On the other hand, as the $S 4$ level increases from 0.3 to 1.0 , the minimum fading depth decreases gradually and reaches as much as $-55 \mathrm{~dB}$. However, when $S 4$ increases beyond the value of 1.1 , the minimum fading depth follows a reverse trend and starts to increase. When $S 4>1.5$, the fading is rarely deeper
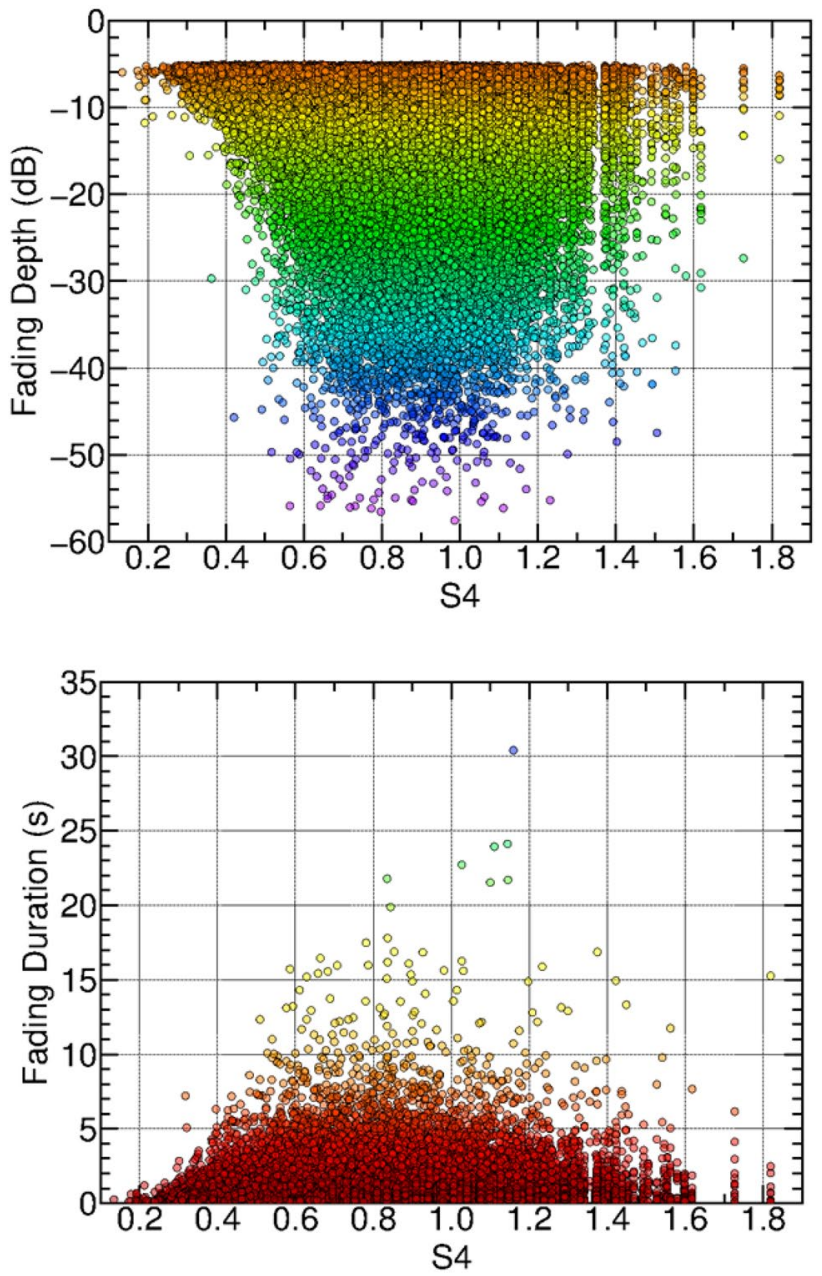

Fig. 8 Distribution of fading depth (top) and fading duration (bottom) in relation to $S 4$ 
than $-30 \mathrm{~dB}$. Consequently, fadings deeper than $-40 \mathrm{~dB}$ are more likely to occur when $S 4$ is between 0.5 and 1.3.

The bottom panel of Fig. 8 presents the distribution of fading duration in relation to $S 4$. Fadings with duration less than $5 \mathrm{~s}$ occur under all levels of scintillation, while these fadings with duration longer than $5 \mathrm{~s}$ are more likely to occur when $S 4$ falls in the range of 0.5-1.4. However, for extremely strong and weak scintillation, the fading duration tends to be short. Furthermore, the maximum duration increases gradually as $S 4$ increases and when it is up to around 1.0, it starts to decrease. The results in Fig. 8 are quite interesting as both the minimum fading depth and maximum fading duration are not linearly proportional to the scintillation levels. For fadings under extremely strong scintillation levels $(S 4>1.4)$, the fading depth is more likely to be higher than $-40 \mathrm{~dB}$ and the duration less than $5 \mathrm{~s}$.

Figure 9 further demonstrates the distribution of various levels of fading depth and duration in relation to $S 4$. Compared with Fig. 8, which mainly demonstrates the trend of fading distribution as a function of $S 4$, the two panels in Fig. 9 illustrate the percentage of fading with respect to the scintillation levels as well as with various levels of depth and duration. It can be seen from the top panel that the fadings with depth from -5 to $-10 \mathrm{~dB}$ account for a large part of all the fadings and that the percentage of these fadings decreases gradually with the increase in $S 4$. By contrast, the percentages of deeper fadings increase when scintillation becomes stronger. From the bottom panel in the figure, it can be observed that fadings with duration shorter than $1 \mathrm{~s}$ seem to account for a large part of fadings for all the scintillation levels, whereas fadings with longer duration probably occur when $S 4 \geq 0.5$, which agrees with the conclusion in the preceding part of the text.

Figures 8 and 9 emphasize the distribution of fading depth and duration for all the detected fadings with respect to scintillation events of different levels. It should be noted that signal intensity fadings do not occur for all scintillation events. The ratio of fading occurrence over scintillation occurrence as a function of $S 4$ is shown in Fig. 10. It can be seen that the ratio increases from around $8 \%$ when $S 4=0.3$ to nearly $80 \%$ when $S 4=0.5$, following which the ratio mostly remains over $80 \%$ for stronger scintillation levels. This indicates a higher probability of the signal intensity suffering from fadings when $S 4$ is over 0.5 .

Next, in this section, the attention is focused on the average fading depth, duration and occurrence for every single scintillation event, i.e., the scintillation within $60 \mathrm{~s}$. It should be noted that only scintillation events with $0.3 \leq S 4 \leq 1.4$ are considered due to the lack of samples when $S 4$ is over 1.4. The occurrence of fadings is counted and the average
Fig. 9 Distribution of fading for various fading depths (top) and fading durations (bottom) with respect to $S 4$
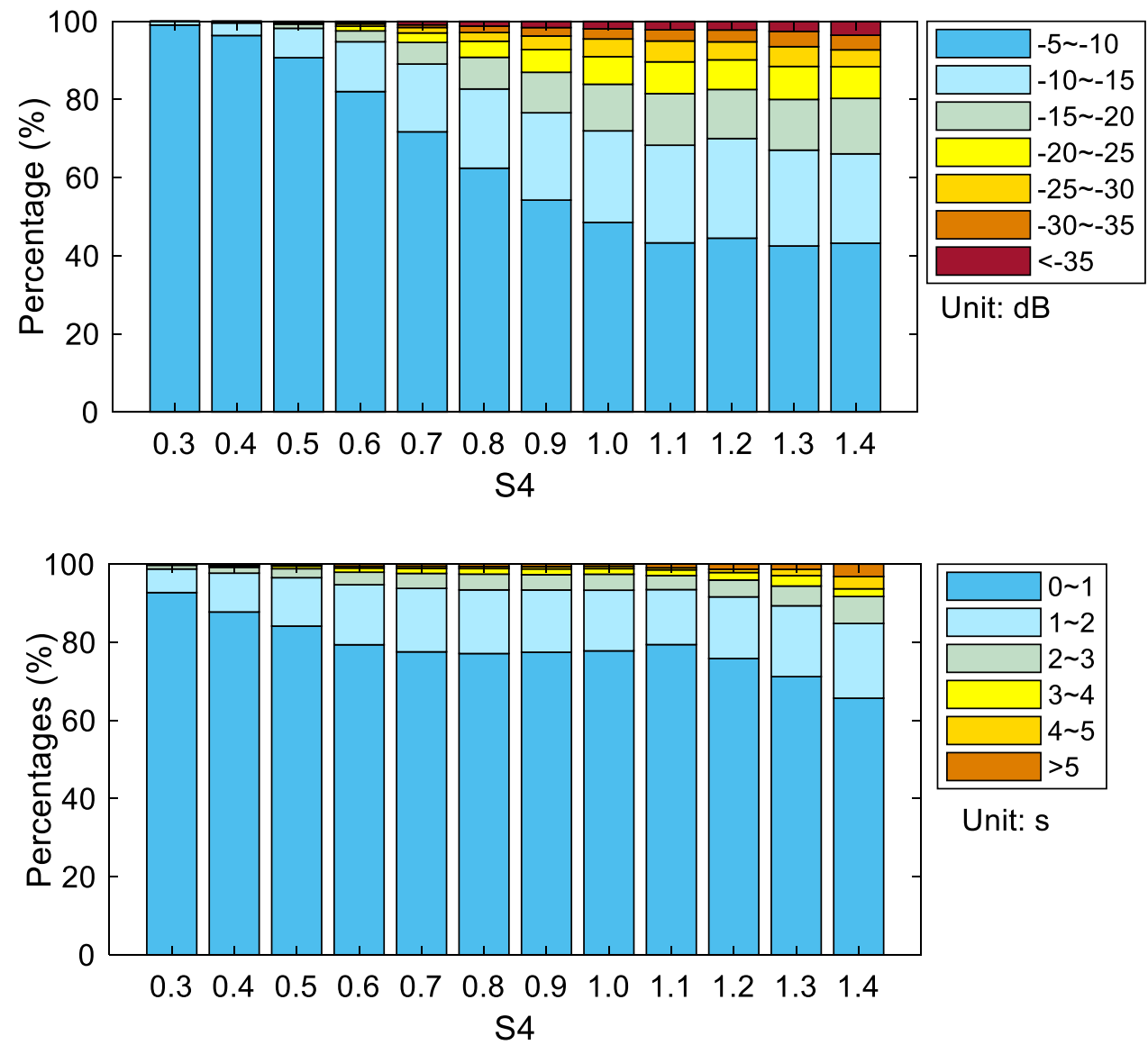


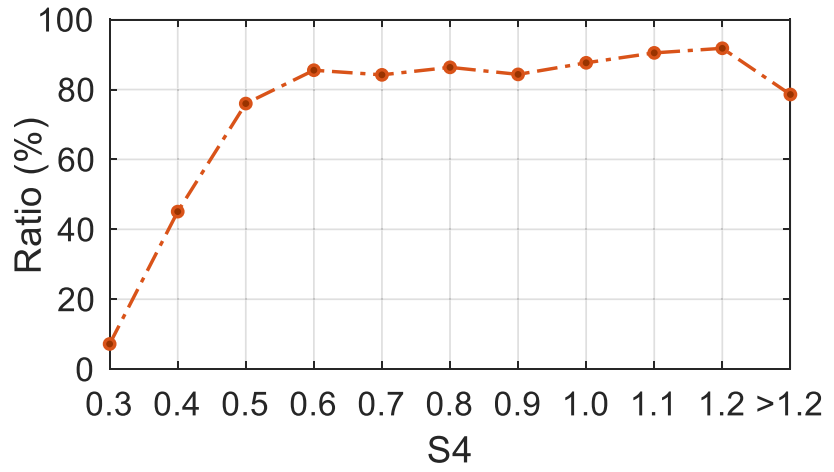

Fig. 10 Ratio of fading occurrence over scintillation events in relation to $S 4$

fading depth, duration and occurrence are, respectively, calculated for all scintillation levels. The variation of the average fading depth is presented in the top panel of Fig. 11. A gradual decrease is observed with the increase in $S 4$. Then the decrease stops and remains at roughly the same level, indicating that the strong scintillation event is likely to correlate with deeper fading generally, but the fading depth does not always decrease. However, in the middle panel, the average fading duration increases as the scintillation becomes more intense. Thus, the fading tends to last longer for strong scintillation on an average, while for the variation of the average fading number in the bottom panel, a slight decrease is observed following the gradual increase. Therefore, it can be concluded that when $S 4$ is over 1.2, the fading occurrence and depth tend to change slightly but the duration probably lasts longer.

The trends observed in Fig. 11 are then fitted to thirdorder polynomial functions, shown by the magenta lines in the figures. The polynomial function is defined as:

$y=a \times(S 4)^{3}+b \times(S 4)^{2}+c \times S 4+d$,

where $y$ can be replaced by $t_{\mathrm{f}}, d_{\mathrm{f}}$ and $N_{\mathrm{f}}$, which is the fading occurrence during $60 \mathrm{~s}$. Table 1 lists the coefficients of the functions. With these functions, the relationship between the general intensity fading depth, duration, occurrence and $S 4$ is mathematically modelled. This is of great importance for a better understanding of the relationship between intensity fading and $S 4$.

\section{Scintillation intensity fading and tracking performance}

The effect of scintillation intensity fading on the receiver tracking loop performance is investigated in this section. As mentioned previously, the PLL tracking error variance is used to indicate the tracking loop performance under scintillation. Figure 12 illustrates an example of increased PLL tracking error variances due to scintillation intensity fading.
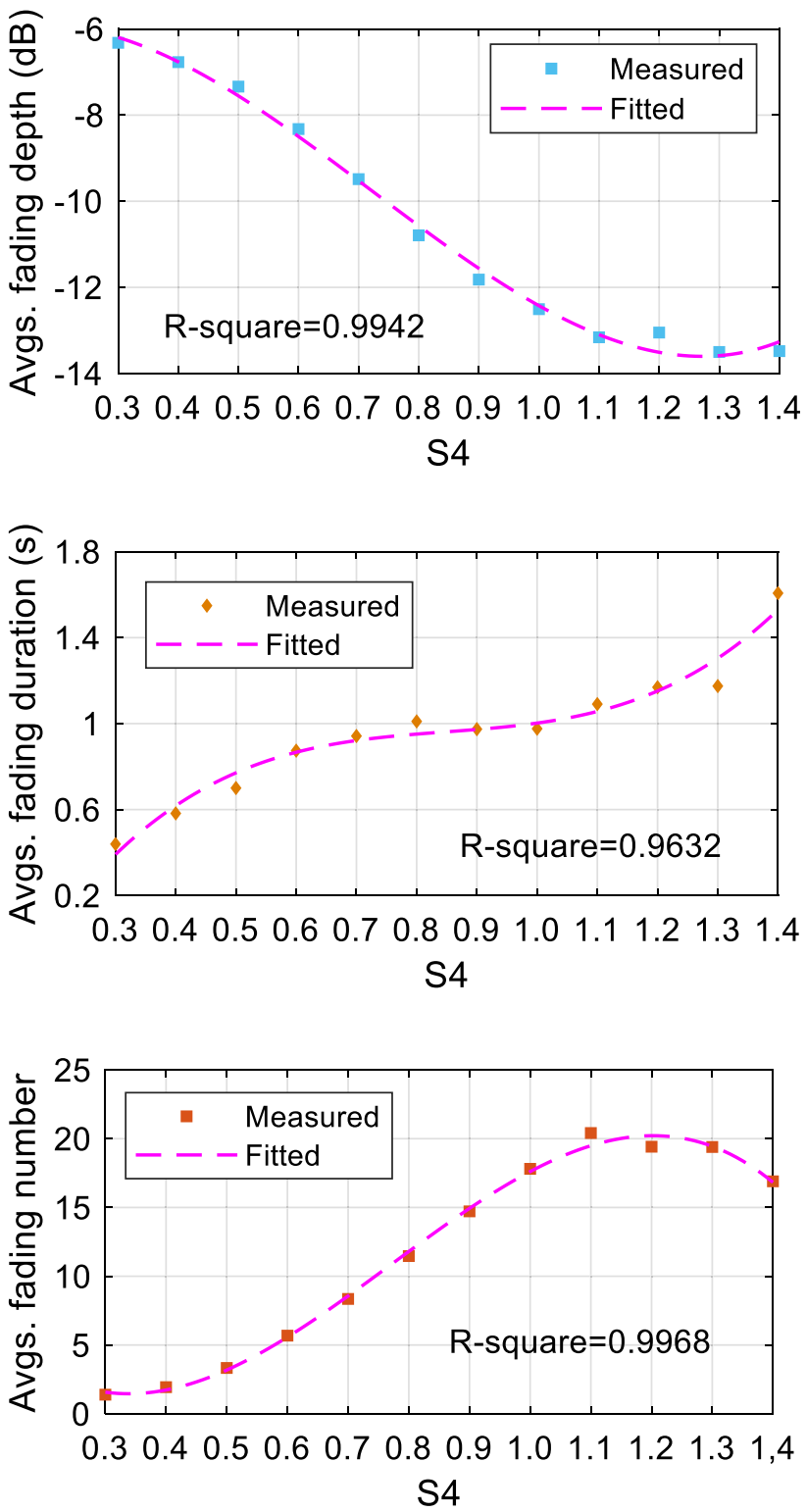

Fig. 11 Variation of average fading depth (top), fading duration (middle) and fading occurrence (bottom) in relation to various $S 4$ levels

In the case of the top two panels, it is considered that there is no scintillation as $S 4$ is lower than 0.1 . The detrended signal intensity is relatively smooth without any obvious fadings. As a result, the tracking error variance is small and might only include the ambient noise effects. On the other hand,

Table 1 Coefficients of the functions for the fitted curves

\begin{tabular}{lcccc}
\hline & $a$ & $b$ & $c$ & $d$ \\
\hline$d_{\mathrm{f}}$ & 11.57 & -25.05 & 7.582 & -6.528 \\
$t_{\mathrm{f}}$ & 2.731 & -6.969 & 6.137 & -0.8966 \\
$N_{\mathrm{f}}$ & -58.15 & 134.4 & -70.98 & 12.34 \\
\hline
\end{tabular}



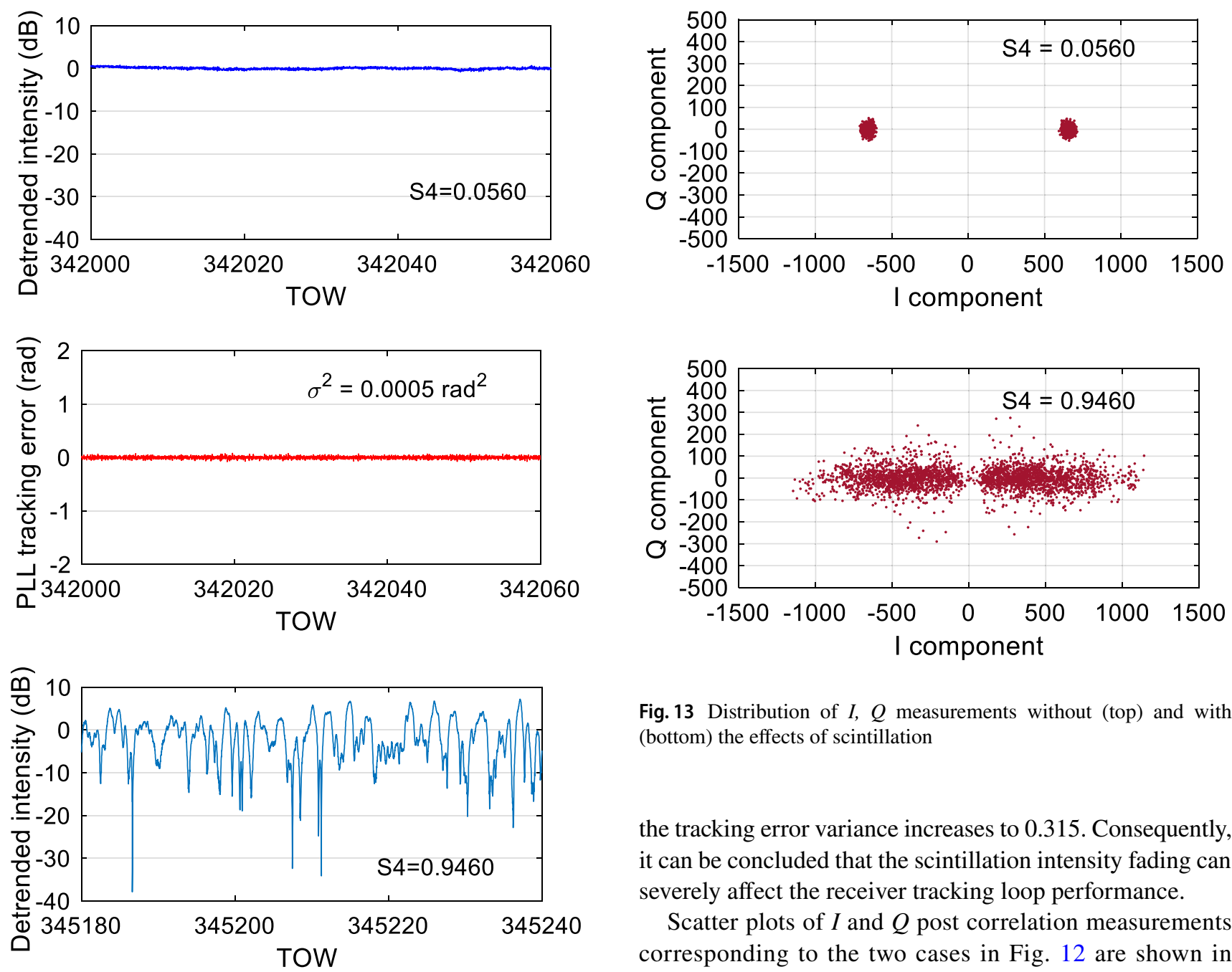

Fig. 13 Distribution of $I, Q$ measurements without (top) and with (bottom) the effects of scintillation

the tracking error variance increases to 0.315 . Consequently, it can be concluded that the scintillation intensity fading can severely affect the receiver tracking loop performance.

Scatter plots of $I$ and $Q$ post correlation measurements corresponding to the two cases in Fig. 12 are shown in Fig. 13. When there is no scintillation in the top panel, the $I / Q$ measurements tend to concentrate on two points, which indicates that the carrier phase is well tracked because almost all the signal intensity is maximum at $I$ measurement (Kaplan and Hegarty 2005). Meanwhile, the noise level, which is indicated by the spread of the clusters, is relatively low. By contrast, the $I$ measurement in the bottom panel presents obvious fluctuations and the noise due to scintillation is evident by the large spread of the points within the two clusters (Kaplan and Hegarty 2005; Parkinson et al. 1996). This further shows the adverse influence of fading on receiver tracking loop performance.

The tracking error variance for every detected fading is

Fig. 12 The increased tracking error variances due to scintillation intensity fading: the variation of the detrended signal intensity and corresponding PLL tracking error variances without (top two panels) and with (bottom two panels) the effects of scintillation

for the case in the bottom two panels, when scintillation is relatively strong with $S 4=0.9460$, the intensity fluctuates significantly and very deep fadings can be obviously found. Furthermore, the tracking errors in this case demonstrate sharper fluctuations corresponding to the deep fadings and calculated using the $I / Q$ post-correlation measurements. These $I / Q$ measurements are raw measurements with the ambient noise maintained. As a result, only fadings detected on the satellite/receiver links over an elevation of $45^{\circ}$ are considered, to minimize the influence of the noise in the analysis. Figure 14 demonstrates the variation of the tracking error variance as a function of fading depth and duration. In the top panel, it can be observed that the tracking error variance may vary significantly for a certain value of 

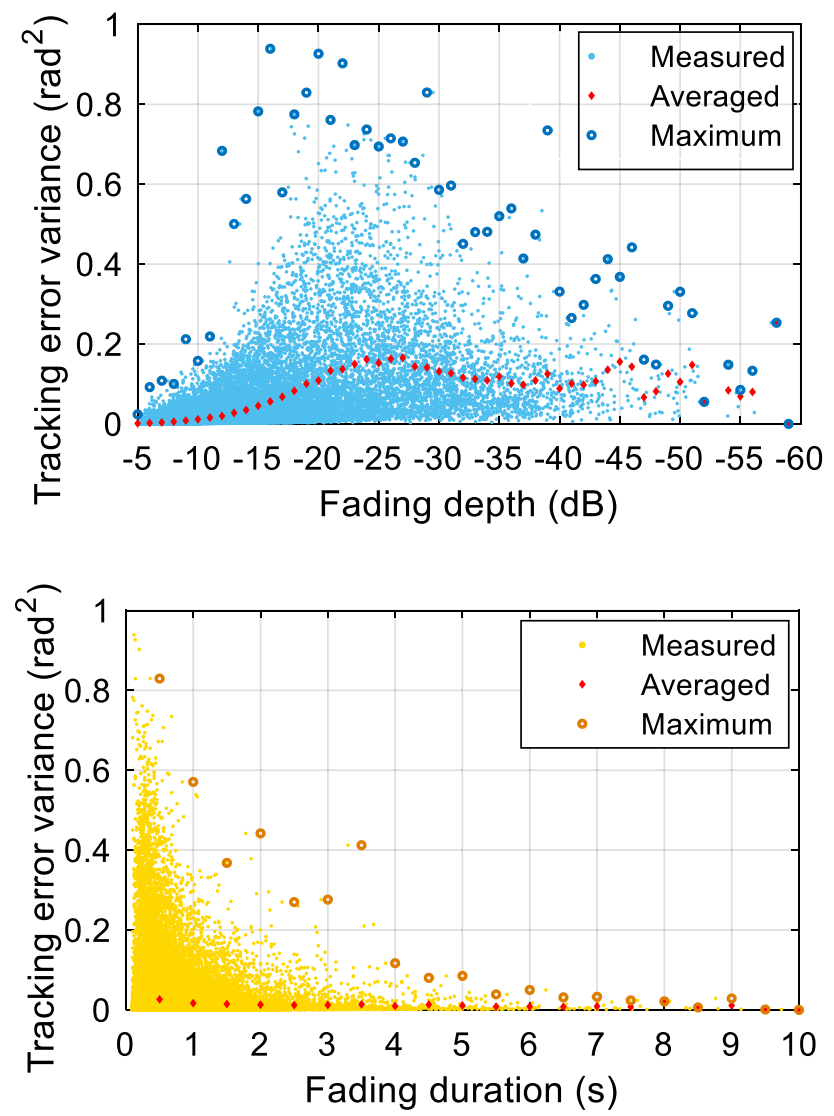

Fig. 14 PLL tracking error variance in relation to fading depth (top) and fading duration (bottom)

fading depth. While for the average tracking error variance, it increases gradually and reaches the peak at around -25 $\mathrm{dB}$, followed by a slight fluctuation around the level of 0.1 $\mathrm{rad}^{2}$. Most of the tracking error variances are lower than $0.4 \mathrm{rad}^{2}$ even when the fading depth is lower than $-45 \mathrm{~dB}$. This might be due to the fact that intensity fading is not the only factor influencing the PLL tracking performance. Regarding the maximum tracking error variance, it increases gradually with the deeper fadings and peaks when the fading depth is around $-20 \mathrm{~dB}$. Then the maximum tracking error variance tends to decrease. This indicates that the fadings with depth around -20 to $-25 \mathrm{~dB}$ are more damaging and more likely to degrade the tracking loop performance. On the other hand, in the bottom panel, the PLL tracking error variance is more likely to be large when the fading duration is shorter than $3 \mathrm{~s}$. The maximum tracking error variance decreases gradually as the fadings last longer. These indicate that shorter fadings tend to increase the PLL tracking errors. It is worth mentioning that only fadings with duration shorter than $10 \mathrm{~s}$ are shown in the figure as there is a lack of samples when the fading duration is longer than $10 \mathrm{~s}$.

To further investigate how the fadings influence the tracking loop performance, the fading speed is defined by

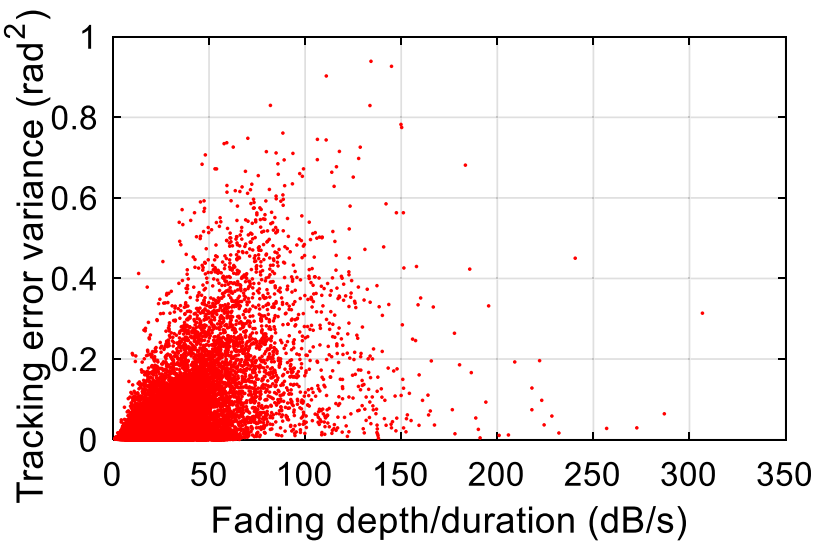

Fig. 15 PLL tracking error variance in relation to fading speed

$v_{\text {fading }}=\frac{\left|d_{\mathrm{f}}\right|}{t_{\mathrm{f}} / 2}(\mathrm{~dB} / \mathrm{s})$. The fadings with a large fading speed are considered sharp fadings. The PLL tracking error variance is then plotted as a function of $v_{\text {fading }}$ in Fig. 15. It can be clearly seen that the overall tracking error variance increases gradually when the fading speed increases. This tendency is reasonable as it is the sharp fadings that really degrade the tracking loop performance in the presence of scintillation. For the tracking error variance in Fig. 15, there is still some ambient receiver noise, hence the tracking error variance is actually a combination of errors due to scintillation intensity fading and ambient noise. However, the focus of this research is to demonstrate the adverse effects of intensity fading on tracking loop performance. Modeling the effects of scintillation signal intensity fading is outside the scope of this study and will be the focus of follow on research.

\section{Conclusion and remarks}

This study focuses on the scintillation intensity fading characterization and investigates the effects of intensity fading on receiver tracking loop performance. The scintillation data analyzed was collected over 3 months during the solar maximum year of 2014 by an ISMR deployed at Presidente Prudente, Brazil, which is a low-latitude station and is subject to severe and frequent ionospheric scintillation.

In the analysis of the daily scintillation from October to December 2014, it can be observed that the occurrence of scintillation is quite frequent at Presidente Prudente. Strong scintillation with $S 4>0.7$ occurs almost every day, with large day-to-day variability. Regarding the intensity fluctuations caused by scintillation, a total number of 144,891 fadings were detected. Most fadings are between -5 and -15 $\mathrm{dB}$, with duration within $1 \mathrm{~s}$. Additionally, an inverse relationship was observed between fading depth and duration. 
Fadings with relatively long duration tend not to be deep. This result is of great importance for a better understanding of the scintillation intensity fading.

By investigating the relationship between $S 4$ and signal intensity fading, it was observed that both the minimum fading depth and the maximum fading duration are not linearly proportional to $S 4$. For fadings caused by extremely strong scintillation, the depth is more likely to be higher than -40 $\mathrm{dB}$ and the duration is less than $5 \mathrm{~s}$. Moreover, it was concluded that fadings with depth from -5 to $-10 \mathrm{~dB}$ account for large parts of all fading events. By contrast, for all the scintillation levels, most fadings are shorter than $1 \mathrm{~s}$. The variation of averaged fading depth, duration and occurrence in relation to $S 4$ for every scintillation event was also studied. Mathematical models were built based on a third-order polynomial function, which bridge the relationship between intensity fading and $S 4$.

The scintillation intensity fading effects on the receiver tracking loop performance were then investigated. By a case study of PLL tracking error variance with and without the effects of scintillation, it was observed that the scintillation intensity fading could severely affect the receiver tracking loop performance. Furthermore, the tracking error variance for every detected fading was calculated and it was concluded that the fadings with depth around $-20 \mathrm{~dB}$ are more damaging and more likely to degrade the tracking loop performance, while with regard to fading duration, the shorter fadings tend to greatly increase the PLL tracking error variance. The fading speed was defined as the ratio of fading depth to duration. It is shown that the tracking error variance increases gradually with the increase in fading speed. As there is still some ambient noise in the intensity measurements, the tracking error variance is not exclusively due to scintillation signal fading. Noise removal and tracking error variance modeling in relation to scintillation intensity fading will be the focus of follow-on research.

Acknowledgements The authors wish to thank the TREASURE project (http://www.treasure-gnss.eu), funded by the European Union's Horizon 2020 Research and Innovation Programme under the Marie Skłodowska-Curie Actions grant agreement No. 722023. Authors also express thanks to João Francisco Galera Monico, Bruno Vani and Italo Tsuchiya in São Paulo State University for offering the professional training and providing the scintillation data.

Open Access This article is distributed under the terms of the Creative Commons Attribution 4.0 International License (http://creativeco mmons.org/licenses/by/4.0/), which permits unrestricted use, distribution, and reproduction in any medium, provided you give appropriate credit to the original author(s) and the source, provide a link to the Creative Commons license, and indicate if changes were made.

\section{References}

Aarons J (1982) Global morphology of ionospheric scintillations. Proc IEEE 70(4):360-378. https://doi.org/10.1109/PROC.1982.12314

Akala AO, Doherty PH, Carrano CS, Valladares CE, Groves KM (2012) Impacts of ionospheric scintillations on GPS receivers intended for equatorial aviation applications. Radio Sci 47:RS4007. https://doi.org/10.1029/2012RS004995

Aquino M, Monico JFG, Dodson AH, Marques H, De Franceschi G, Alfonsi L, Romano V, Andreotti M (2009) Improving the GNSS positioning stochastic model in the presence of ionospheric scintillation. J Geod 83(10):953-966. https://doi.org/10.1007/s0019 0-009-0313-6

Basu S, MacKenzie E, Basu S (1988) Ionospheric constraints on VHF/ UHF communications links during solar maximum and minimum periods. Radio Sci 23(3):363-378. https://doi.org/10.1029/RS023 i003p00363

Conker RS, El-Arini MB, Hegarty CJ, Hsiao T (2003) Modeling the effects of ionospheric scintillation on GPS/satellite-based augmentation system availability. Radio Sci 38(1):1-1-1-23. https:// doi.org/10.1029/2000RS002604

Davies K (1990) Ionospheric radio. Peter Perrgrinus Ltd., London

Forte B (2012) Analysis of the PLL phase error in presence of simulated ionospheric scintillation events. Radio Sci 47:RS3006. https ://doi.org/10.1029/2011RS004790

Fortes LPS, Lin T, Lachapelle G (2015) Effects of the 2012-2013 solar maximum on GNSS signals in Brazil. GPS Solut 19(2):309-319. https://doi.org/10.1007/s10291-014-0389-1

Groves KM, Basu S, Quinn JM, Pedersen TR, Falinski K, Brown A, Silva R, Ning P (2000) A comparison of GPS performance in a scintillation environment at Ascension Island. In: Proceedings of ION GPS 2000. Institute of Navigation, Salt Lake City, UT, 19-22 September 2000, pp 672-679

Hegarty C, El-Arini MB, Kim T, Ericson S (2001) Scintillation modeling for GPS-wide area augmentation system receivers. Radio Sci 36(5):1221-1231. https://doi.org/10.1029/1999RS002425

Jiao Y, Dongyang X, Morton Y, Rino C (2016) Equatorial scintillation amplitude fading characteristics across the GPS frequency bands. J Inst Navig 63:267-281. https://doi.org/10.1002/navi.146

Kai G, Yan Z, Yang L, Jinling W, Chunxi Z, Yanbo Z (2017) Study of ionospheric scintillation characteristics in Australia with GNSS during 2011-2015. Adv Space Res 59(12):2909-2922. https://doi. org/10.1016/j.asr.2017.03.007

Kaplan ED, Hegarty C (2005) Understanding GPS: principles and applications, 2nd edn. Artech House, Norwood

Moraes ADO, Rodrigues FDS, Perrella WJ, Paula ERD (2012) Analysis of the characteristics of low-latitude GPS amplitude scintillation measured during solar maximum conditions and implications for receiver performance. Surv Geophys 33(5):1107-1131. https ://doi.org/10.1007/s10712-011-9161-z

Mushini SC, Jayachandran PT, Langley RB, MacDougall JW, Pokhotelov D (2012) Improved amplitude- and phase-scintillation indices derived from wavelet detrended high-latitude GPS data. GPS Solut 16(3):363-373. https://doi.org/10.1007/s10291-011-0238-4

Parkinson B, Spilker J, Axelrad P, Enge P (1996) Global positioning system: theory and applications, vol II. American Institute of Aeronautics and Astronautics Inc, Washington

Seo J, Walter T, Chiou TY, Enge P (2016) Characteristics of deep GPS signal fading due to ionospheric scintillation for aviation receiver design. Radio Sci 44(1):1-10. https://doi.org/10.1029/2008R S004077

Spogli L, Alfonsi L, Romano V, De Franceschi G, Monico JFG, Shimabukuro MH, Bougard B, Aquino M (2013) Assessing the GNSS scintillation climate over Brazil under increasing solar 
activity. J Atmos Solar Terr Phys 105-106:199-206. https://doi. org/10.1016/j.jastp.2013.10.003

Sreeja V, Aquino M, Elmas ZG (2011) Impact of ionospheric scintillation on GNSS receiver tracking performance over Latin America: introducing the concept of tracking jitter variance maps. Space Weather 9(10):1-6. https://doi.org/10.1029/2011SW000707

Sreeja V, Aquino M, Elmas ZG, Forte B (2012) Correlation analysis between ionospheric scintillation levels and receiver tracking performance. Space Weather. https://doi.org/10.1029/2012SW0007 69

Van Dierendonck AJ, Arbesser-Rastburg B (2004) Measuring Ionospheric scintillation in the equatorial region over Africa, including measurements from SBAS geostationary satellite signals. In: Proceedings of ION GNSS 2004. Institute of Navigation, Long Beach, CA, 21-24 September 2004, pp 316-324

Van Dierendonck AJ, Klobuchar J, Hua Q (1993) Ionospheric scintillation monitoring using commercial single frequency C/A code receivers. In: Proceedings of ION GPS 1993. Institute of Navigation, Salt Lake City, UT, 22-24 September 1993, pp 1333-1342

Vani BC, Shimabukuro MH, Monico JFG (2017) Visual exploration and analysis of ionospheric scintillation monitoring data: the ISMR query tool. Comput Geosci 104:125-134. https://doi. org/10.1016/j.cageo.2016.08.022

Yeh KC, Liu CH (1982) Radio wave scintillations in the ionosphere. Proc IEEE 70(4):324-360. https://doi.org/10.1109/ PROC.1982.12313

Publisher's Note Springer Nature remains neutral with regard to jurisdictional claims in published maps and institutional affiliations.

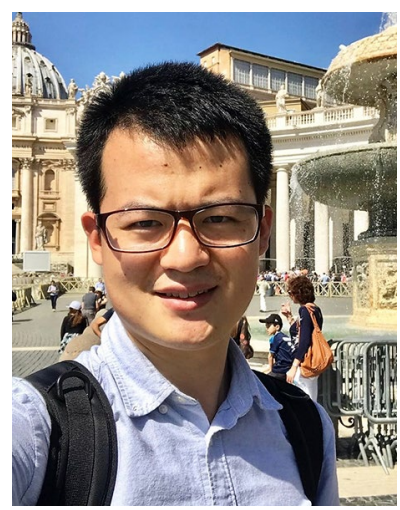

Kai Guo is a Marie SkłodowskaCurie research fellow at the Nottingham Geospatial Institute of the University of Nottingham in the UK, within the TREASURE project funded by the European Union's Horizon 2020 Research and Innovation Programme. $\mathrm{He}$ concentrates on developing novel GNSS receiver tracking models and scintillation mitigation tools, aiming to improve the receiver tracking performance under ionospheric scintillation.

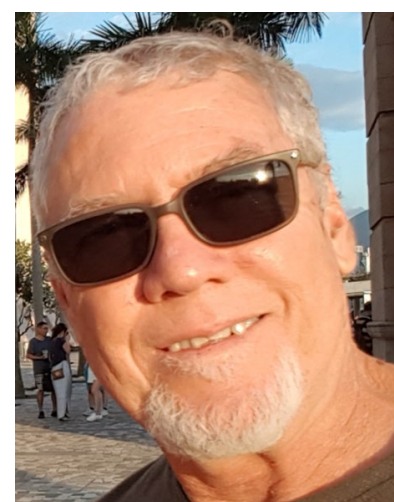

Marcio Aquino is an Associate Professor at the Nottingham Geospatial Institute of the University of Nottingham in the UK. He pioneered the deployment of ionospheric scintillation and TEC monitoring receivers in Northern Europe in 2001. His research has focused on ionospheric effects on GNSS, including system vulnerability to ionospheric disturbances and relevant counter measures.

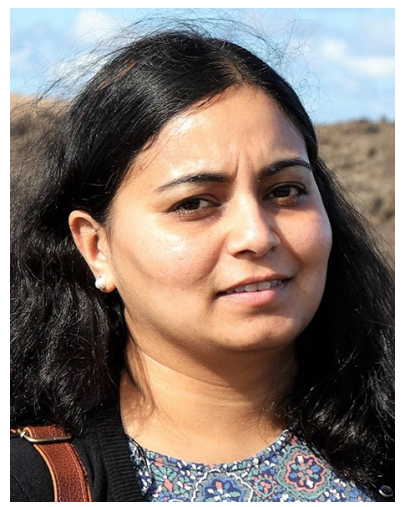

Sreeja Vadakke Veettil is a senior research fellow at the Nottingham Geospatial Institute of the University of Nottingham in the $\mathrm{UK}$, having been involved in European Commission, European Space Agency and UK research council funded projects. Her research focuses on assessing the effects of space weather on GNSS receivers and quantifying positioning errors aiming to improve the modeling of scintillation and to develop scintillation mitigation tools. 